$10-20-2016$

\title{
Software Engineering for Science
}

Jeffrey C. Carver

University of Alabama - Tuscaloosa

Neil P. Chue Hong

University of Edinburgh

George K. Thiruvathukal

Loyola University Chicago, gkt@cs.luc.edu

Follow this and additional works at: https://ecommons.luc.edu/cs_facpubs

Part of the Numerical Analysis and Scientific Computing Commons, and the Software Engineering Commons

\section{Recommended Citation}

Jeffrey C. Carver, Neil Chue P. Hong, and George K. Thiruvathukal (editors), Software Engineering for Science, Taylor and Francis/CRC Press.

This Book Chapter is brought to you for free and open access by the Faculty Publications and Other Works by Department at Loyola eCommons. It has been accepted for inclusion in Computer Science: Faculty Publications and Other Works by an authorized administrator of Loyola eCommons. For more information, please contact ecommons@luc.edu.

Copyright (c) 2016, Taylor and Francis, CRC Press. 


\section{SOFTWARE \\ ENGINEERING \\ FOR SCIENCE}




\title{
Chapman \& Hall/CRC \\ Computational Science Series \\ SERIES EDITOR
}

\author{
Horst Simon \\ Deputy Director \\ Lawrence Berkeley National Laboratory \\ Berkeley, California, U.S.A.
}

\section{PUBLISHED TITLES}

COMBINATORIAL SCIENTIFIC COMPUTING

Edited by Uwe Naumann and Olaf Schenk

CONTEMPORARY HIGH PERFORMANCE COMPUTING: FROM PETASCALE

TOWARD EXASCALE

Edited by Jeffrey S. Vetter

CONTEMPORARY HIGH PERFORMANCE COMPUTING: FROM PETASCALE

TOWARD EXASCALE, VOLUME TWO

Edited by Jeffrey S. Vetter

DATA-INTENSIVE SCIENCE

Edited by Terence Critchlow and Kerstin Kleese van Dam

THE END OF ERROR: UNUM COMPUTING

John L. Gustafson

FROM ACTION SYSTEMS TO DISTRIBUTED SYSTEMS: THE REFINEMENT APPROACH

Edited by Luigia Petre and Emil Sekerinski

FUNDAMENTALS OF MULTICORE SOFTWARE DEVELOPMENT

Edited by Victor Pankratius, Ali-Reza Adl-Tabatabai, and Walter Tichy

FUNDAMENTALS OF PARALLEL MULTICORE ARCHITECTURE

Yan Solihin

THE GREEN COMPUTING BOOK: TACKLING ENERGY EFFICIENCY AT LARGE SCALE

Edited by Wu-chun Feng

GRID COMPUTING: TECHNIQUES AND APPLICATIONS

Barry Wilkinson

HIGH PERFORMANCE COMPUTING: PROGRAMMING AND APPLICATIONS

John Levesque with Gene Wagenbreth

HIGH PERFORMANCE PARALLEL I/O

Prabhat and Quincey Koziol 


\title{
PUBLISHED TITLES CONTINUED
}

\author{
HIGH PERFORMANCE VISUALIZATION: \\ ENABLING EXTREME-SCALE SCIENTIFIC INSIGHT \\ Edited by E. Wes Bethel, Hank Childs, and Charles Hansen
}

INDUSTRIAL APPLICATIONS OF HIGH-PERFORMANCE COMPUTING:

BEST GLOBAL PRACTICES

Edited by Anwar Osseyran and Merle Giles

INTRODUCTION TO COMPUTATIONAL MODELING USING C AND

OPEN-SOURCE TOOLS

José M Garrido

INTRODUCTION TO CONCURRENCY IN PROGRAMMING LANGUAGES

Matthew J. Sottile, Timothy G. Mattson, and Craig E Rasmussen

INTRODUCTION TO ELEMENTARY COMPUTATIONAL MODELING: ESSENTIAL CONCEPTS, PRINCIPLES, AND PROBLEM SOLVING

José M. Garrido

INTRODUCTION TO HIGH PERFORMANCE COMPUTING FOR SCIENTISTS

AND ENGINEERS

Georg Hager and Gerhard Wellein

INTRODUCTION TO REVERSIBLE COMPUTING

Kalyan S. Perumalla

INTRODUCTION TO SCHEDULING

Yves Robert and Frédéric Vivien

INTRODUCTION TO THE SIMULATION OF DYNAMICS USING SIMULINK ${ }^{\circledR}$

Michael A. Gray

PEER-TO-PEER COMPUTING: APPLICATIONS, ARCHITECTURE, PROTOCOLS, AND CHALLENGES

Yu-Kwong Ricky Kwok

PERFORMANCE TUNING OF SCIENTIFIC APPLICATIONS

Edited by David Bailey, Robert Lucas, and Samuel Williams

PETASCALE COMPUTING: ALGORITHMS AND APPLICATIONS

Edited by David A. Bader

PROCESS ALGEBRA FOR PARALLEL AND DISTRIBUTED PROCESSING

Edited by Michael Alexander and William Gardner

SCIENTIFIC DATA MANAGEMENT: CHALLENGES, TECHNOLOGY, AND DEPLOYMENT

Edited by Arie Shoshani and Doron Rotem

SOFTWARE ENGINEERING FOR SCIENCE

Edited by Jeffrey C. Carver, Neil P. Chue Hong, and George K. Thiruvathukal 
- 


\title{
SOFTWARE ENGINEERING FOR SCIENCE
}

\author{
Edited by \\ Jeffrey C. Carver \\ University of Alabama, USA \\ Neil P. Chue Hong \\ University of Edinburgh, UK \\ George K. Thiruvathukal \\ Loyola University Chicago, Chicago, Illinois
}

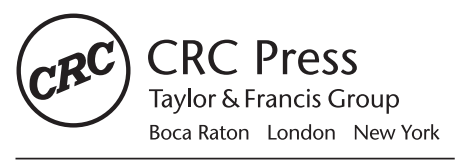

CRC Press is an imprint of the

Taylor \& Francis Group, an informa business

A CHAPMAN \& HALL BOOK 
MATLAB ${ }^{\circ}$ is a trademark of The MathWorks, Inc. and is used with permission. The MathWorks does not warrant the accuracy of the text or exercises in this book. This book's use or discussion of MATLAB ${ }^{\circ}$ soft- $^{\circ}$ ware or related products does not constitute endorsement or sponsorship by The MathWorks of a particular pedagogical approach or particular use of the MATLAB ${ }^{\circ}$ software.

\section{CRC Press}

Taylor \& Francis Group

6000 Broken Sound Parkway NW, Suite 300

Boca Raton, FL 33487-2742

(c) 2017 by Taylor \& Francis Group, LLC

CRC Press is an imprint of Taylor \& Francis Group, an Informa business

No claim to original U.S. Government works

Printed on acid-free paper

Version Date: 20160817

International Standard Book Number-13: 978-1-4987-4385-3 (Hardback)

This book contains information obtained from authentic and highly regarded sources. Reasonable efforts have been made to publish reliable data and information, but the author and publisher cannot assume responsibility for the validity of all materials or the consequences of their use. The authors and publishers have attempted to trace the copyright holders of all material reproduced in this publication and apologize to copyright holders if permission to publish in this form has not been obtained. If any copyright material has not been acknowledged please write and let us know so we may rectify in any future reprint.

Except as permitted under U.S. Copyright Law, no part of this book may be reprinted, reproduced, transmitted, or utilized in any form by any electronic, mechanical, or other means, now known or hereafter invented, including photocopying, microfilming, and recording, or in any information storage or retrieval system, without written permission from the publishers.

For permission to photocopy or use material electronically from this work, please access www.copyright. com (http://www.copyright.com/) or contact the Copyright Clearance Center, Inc. (CCC), 222 Rosewood Drive, Danvers, MA 01923, 978-750-8400. CCC is a not-for-profit organization that provides licenses and registration for a variety of users. For organizations that have been granted a photocopy license by the CCC, a separate system of payment has been arranged.

Trademark Notice: Product or corporate names may be trademarks or registered trademarks, and are used only for identification and explanation without intent to infringe.

\section{Library of Congress Cataloging-in-Publication Data}

Names: Carver, Jeffrey, editor. | Hong, Neil P. Chue, editor. |

Thiruvathukal, George K. (George Kuriakose), editor.

Title: Software engineering for science / edited by Jeffrey Carver, Neil P.

Chue Hong, and George K. Thiruvathukal.

Description: Boca Raton : Taylor \& Francis, CRC Press, 2017. | Series:

Computational science series ; 30 | Includes bibliographical references and index.

Identifiers: LCCN 2016022277 | ISBN 9781498743853 (alk. paper)

Subjects: LCSH: Science--Data processing. | Software engineering.

Classification: LCC Q183.9 .S74 2017 | DDC 005.1--dc23

LC record available at https://lccn.loc.gov/2016022277

Visit the Taylor \& Francis Web site at

http://www.taylorandfrancis.com

and the CRC Press Web site at

http://www.crcpress.com 


\section{Contents}

List of Figures $\quad$ Xv

List of Tables $\quad$ xvii

About the Editors $\quad$ xix

List of Contributors $\quad$ xxi

Acknowledgments $\quad$ xxv

Introduction $\quad$ xxvii

1 Software Process for Multiphysics Multicomponent Codes 1

Anshu Dubey, Katie Antypas, Ethan Coon, and Katherine Riley

1.1 Introduction . . . . . . . . . . . . . . . 2

1.2 Lifecycle . . . . . . . . . . . . . . . . 3

1.2.1 Development Cycle . . . . . . . . . . . . . . . 4

1.2.2 Verification and Validation . . . . . . . . . . . 4

1.2.3 Maintenance and Extensions . . . . . . . . . . . 6

1.2.4 Performance Portability . . . . . . . . . . . . 7

1.2.5 Using Scientific Software . . . . . . . . . . . . . . 7

1.3 Domain Challenges . . . . . . . . . . . . . . . . 8

1.4 Institutional and Cultural Challenges _. . . . . . . . . 9

1.5 Case Studies . . . . . . . . . . . . . . . . . . . . . 12

$1.5 .1 \quad$ FLASH $\ldots \ldots \ldots \ldots \ldots \ldots \ldots$

1.5.1.1 Code Design . . . . . . . . . . . . . 12

1.5.1.2 Verification and Validation . . . . . . . . 14

1.5.1.3 Software Process . . . . . . . . . . . . . 16

1.5.1.4 Policies . . . . . . . . . . . . . . . . . 18

$1.5 .2 \quad$ Amanzi/ATS . . . . . . . . . . . . . . . . . 19

1.5.2.1 Multiphysics Management through Arcos . . 20

1.5.2.2 Code Reuse and Extensibility . . . . . . . . . 21

1.5.2.3 Testing . . . . . . . . . . . . . . 21

1.5.2.4 Performance Portability . . . . . . . . . . 22 
1.6 Generalization ............... . . 23

1.7 Additional Future Considerations . . . . . . . . . . . . . 25

2 A Rational Document Driven Design Process for Scientific Software

W. Spencer Smith

2.1 Introduction . . . . . . . . . . . . . . . . . . . 27

2.2 A Document Driven Method .............. . 31

2.2.1 Problem Statement . . . . . . . . . . . . . . . 32

2.2.2 Development Plan . . . . . . . . . . . . . . 33

2.2.3 Software Requirements Specification (SRS) . . . . . . 34

2.2.4 Verification and Validation (V\&V) Plan and Report . 35

2.2.5 Design Specification ............ . . . . 37

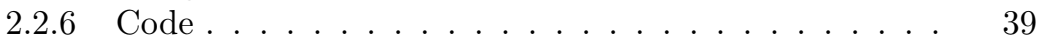

2.2 .7 User Manual . . . . . . . . . . . . . . . . . . . 40

2.2.8 Tool Support . . . . . . . . . . . . . . . . . 41

2.3 Example: Solar Water Heating Tank . . . . . . . . . . . . . . 41

2.3.1 Software Requirements Specification (SRS) . . . . . . 42

2.3.2 Design Specification ............. . . 45

2.4 Justification . . . . . . . . . . . . . . . . . . . . . . . 47

2.4.1 Comparison between CRAN and Other Communities . 48

2.4.2 Nuclear Safety Analysis Software Case Study . . . . . 49

2.5 Concluding Remarks . . . . . . . . . . . . . . 50

3 Making Scientific Software Easier to Understand, Test, and Communicate through Software Engineering

Matthew Patrick

3.1 Introduction . . . . . . . . . . . . . . . . . . 54

3.2 Case Studies . . . . . . . . . . . . . . . . . . 56

3.3 Challenges Faced by the Case Studies . . . . . . . . . . 56

3.3.1 Intuitive Testing . . . . . . . . . . . . . . 60

3.3.2 Automating Tests . . . . . . . . . . . . . 62

3.3.3 Legacy Code ................... 64

3.3.4 Summary ................. . . 66

3.4 Iterative Hypothesis Testing . . . . . . . . . . . . . . 66

3.4.1 The Basic SEIR Model . . . . . . . . . . . . . . 67

3.4.2 Experimental Methodology . . . . . . . . . . 68

3.4.3 Initial Hypotheses . . . . . . . . . . . . . . . . . 69

3.4.3.1 Sanity Checks . . . . . . . . . . . 69

3.4.3.2 Metamorphic Relations .......... 70

3.4.3.3 Mathematical Derivations . . . . . . . . . 71

3.4.4 Exploring and Refining the Hypotheses . . . . . . . 71

3.4.4.1 Complexities of the Model . . . . . . . . . 72

3.4.4.2 Complexities of the Implementation . . . . . 73

3.4.4.3 Issues Related to Numerical Precision . . . . 74 
Contents ix

3.4.5 Summary ................... . . 75

3.5 Testing Stochastic Software Using Pseudo-Oracles . . . . . . 77

3.5.1 The Huánglóngbìng SECI Model . . . . . . . . . . . . 78

3.5.2 Searching for Differences . . . . . . . . . . . . 80

3.5.3 Experimental Methodology . . . . . . . . . . . 82

3.5.4 Differences Discovered . . . . . . . . . . . . . . . . 82

3.5.5 Comparison with Random Testing . . . . . . . . . . 86

3.5.6 Summary .................. . . 87

3.6 Conclusions . . . . . . . . . . . . . . . . . . . . . . 87

3.7 Acknowledgments ................. 88

4 Testing of Scientific Software: Impacts on Research Credibility, Development Productivity, Maturation, and Sustainability

Roscoe A. Bartlett, Anshu Dubey, Xiaoye Sherry Li, J. David Moulton, James M. Willenbring, and Ulrike Meier Yang

4.1 Introduction . . . . . . . . . . . . . . . . . . . 90

4.2 Testing Terminology . . . . . . . . . . . . . . . . . . . . . 92

4.2.1 Granularity of Tests . . . . . . . . . . . . . . . 92

4.2.2 Types of Tests . . . . . . . . . . . . . . 93

4.2.3 Organization of Tests . . . . . . . . . . . . . . 94

4.2.4 Test Analysis Tools . . . . . . . . . . . . . . . 95

4.3 Stakeholders and Team Roles for CSE Software Testing . . . 95

4.3.1 Stakeholders ... . . . . . . . . . . . . 95

4.3.2 Key Roles in Effective Testing . . . . . . . . . . . . . 96

4.3.3 Caveats and Pitfalls . . . . . . . . . . . . . . . . . 97

4.4 Roles of Automated Software Testing in CSE Software . . . 98

4.4.1 Role of Testing in Research . . . . . . . . . . . . . 98

4.4.2 Role of Testing in Development Productivity . . . . . 100

4.4.3 Role of Testing in Software Maturity and Sustainability 102

4.5 Challenges in Testing Specific to CSE . . . . . . . . . . 103

4.5.1 Floating-Point Issues and Their Impact on Testing . . 103

4.5.2 Scalability Testing . . . . . . . . . . . . . 105

4.5.3 Model Testing . . . . . . . . . . . . . . 107

4.6 Testing Practices . . . . . . . . . . . . . . . . . . . . . 109

4.6.1 Building a Test Suite for CSE Codes . . . . . . . . . . 110

4.6.2 Evaluation and Maintenance of a Test Suite . . . . . . 112

4.6.3 An Example of a Test Suite . . . . . . . . . . . . . 113

4.6.4 Use of Test Harnesses . . . . . . . . . . . . . . . 114

4.6.5 Policies .................. 116

4.7 Conclusions . . . . . . . . . . . . . . . . . . . . 117

4.8 Acknowledgments .................. . . 118 
5 Preserving Reproducibility through Regression Testing

Daniel Hook

5.1 Introduction . . . . . . . . . . . . . . . . . . . 119

5.1 .1 Other Testing Techniques . . . . . . . . . . . . 120

5.1 .2 Reproducibility . . . . . . . . . . . . . . . . . . 121

5.1 .3 Regression Testing . . . . . . . . . . . . . . . . . . 122

5.2 Testing Scientific Software . . . . . . . . . . . . . . 123

5.2.1 The Oracle and Tolerance Problems . . . . . . . . . 123

5.2.1.1 Sensitivity Testing . . . . . . . . . . . . 125

5.2.2 Limitations of Regression Testing . . . . . . . . . . 125

5.3 Regression Testing at ESG . . . . . . . . . . . . . . 126

5.3 .1 Building the Tools . . . . . . . . . . . . . . 127

5.3.1.1 Key Lesson . . . . . . . . . . . . . . . . . 129

$5.3 .2 \quad$ Selecting the Tests . . . . . . . . . . . . . . . . . 129

5.3.2.1 Key Lessons . . . . . . . . . . . . . . 130

5.3 .3 Evaluating the Tests . . . . . . . . . . . . . . . 130

5.3.3.1 Key Lessons . . . . . . . . . . . . . 130

5.3 .4 Results . . . . . . . . . . . . . . . . . 131

5.4 Conclusions and Future Work _. . . . . . . . . . . . 132

6 Building a Function Testing Platform for Complex Scientific Code

Dali Wang, Zhuo Yao, and Frank Winkler

6.1 Introduction . . . . . . . . . . . . . . . . . 135

6.2 Software Engineering Challenges for Complex Scientific Code 136

6.3 The Purposes of Function Unit Testing for Scientific Code . 136

6.4 Generic Procedure of Establishing Function Unit Testing for

Large-Scale Scientific Code . . . . . . . . . . . . . . . . 137

6.4.1 Software Analysis and Testing Environment

Establishment . . . . . . . . . . . . . . . . 138

6.4.2 Function Unit Test Module Generation . . . . . . . . 139

6.4.3 Benchmark Test Case Data Stream Generation Using Variable Tracking and Instrumentation . . . . . . . . . 139

6.4.4 Function Unit Module Validation . . . . . . . . . . . . 139

6.5 Case Study: Function Unit Testing for the ACME Model . . 140

6.5.1 ACME Component Analysis and Function Call-Tree

Generation . . . . . . . . . . . . . . 140

6.5.2 Computational Characteristics of ACME Code . . . . 141

6.5.3 A Function Unit Testing Platform for ACME Land Model . . . . . . . . . . . . . . . . . . . . . 144

6.5.3.1 System Architecture of ALM Function Test Framework . . . . . . . . . . . . 144

6.5.3.2 Working Procedure of the ALM Function Test Framework . . . . . . . . . . 146

6.6 Conclusion . . . . . . . . . . . . . . . . . . . 148 
Upulee Kanewala, Anders Lundgren, and James M. Bieman

7.1 Introduction . . . . . . . . . . . . . . . . . . . . . 150

7.2 The Oracle Problem in Scientific Software . . . . . . . . . . . 152

7.3 Metamorphic Testing for Testing Scientific Software . . . . . 154

7.3.1 Metamorphic Testing .............. 154

7.3.2 Applications of MT for Scientific Software Testing . . 155

7.4 MRpred: Automatic Prediction of Metamorphic Relations . . 157

7.4.1 Motivating Example . . . . . . . . . . . . . . 157

7.4.2 Method Overview ................ 158

7.4.3 Function Representation . . . . . . . . . . . . 160

7.4 .4 Graph Kernels . . . . . . . . . . . . . . . . . . 161

7.4.4.1 The Random Walk Kernel . . . . . . . . . . 161

7.4.5 Effectiveness of MRpred . . . . . . . . . . . 162

7.5 Case Studies . . . . . . . . . . . . . . . . . . . 162

7.5.1 Code Corpus . . . . . . . . . . . . . . 163

7.5.2 Metamorphic Relations ............. 165

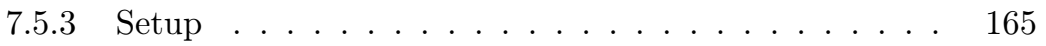

7.6 Results ....................... 167

7.6.1 Overall Fault Detection Effectiveness . . . . . . . . . . 167

7.6.2 Fault Detection Effectiveness across MRs . . . . . . . 168

7.6.3 Effectiveness of Detecting Different Fault Categories . 171

7.7 Conclusions and Future Work . . . . . . . . . . . . . . . 172

8 Evaluating Hierarchical Domain-Specific Languages for

Computational Science: Applying the Sprat Approach

to a Marine Ecosystem Model

Arne N. Johanson, Wilhelm Hasselbring, Andreas Oschlies, and Boris

Worm

8.1 Motivation .................... 176

8.2 Adapting Domain-Specific Engineering Approaches for

Computational Science . . . . . . . . . . . . . . . 177

8.3 The Sprat Approach: Hierarchies of Domain-Specific Languages 179

8.3.1 The Architecture of Scientific Simulation Software . . 179

8.3.2 Hierarchies of Domain-Specific Languages . . . . . . . 181

8.3.2.1 Foundations of DSL Hierarchies . . . . . . 182

8.3.2.2 An Example Hierarchy . . . . . . . . . . . 183

8.3.3 Applying the Sprat Approach . . . . . . . . . . . 185

8.3.3.1 Separating Concerns . . . . . . . . . . 186

8.3.3.2 Determining Suitable DSLs . . . . . . . . . 186

8.3.3.3 Development and Maintenance . . . . . . . 188

8.3.4 Preventing Accidental Complexity . . . . . . . . . . 189

8.4 Case Study: Applying Sprat to the Engineering of a Coupled

Marine Ecosystem Model . . . . . . . . . . . . . . . . . 190

8.4.1 The Sprat Marine Ecosystem Model . . . . . . . . . . 190 
8.4.2 The Sprat PDE DSL . . . . . . . . . . . . . . . . . . 191

8.4.3 The Sprat Ecosystem DSL . . . . . . . . . . . . . . . . 192

8.4.4 The Ansible Playbook DSL . . . . . . . . . . . . . . 192

8.5 Case Study Evaluation . . . . . . . . . . . . . . . . . 193

8.5.1 Data Collection . . . . . . . . . . . . . . . . . 193

8.5.2 Analysis Procedure . . . . . . . . . . . . . 195

8.5.3 Results from the Expert Interviews . . . . . . . . . . 195

8.5.3.1 Learning Material for DSLs . . . . . . . . . . 195

8.5.3.2 Concrete Syntax: Prescribed vs. Flexible

Program Structure . . . . . . . . . . 196

8.5.3.3 Internal vs. External Implementation . . . . 197

8.6 Conclusions and Lessons Learned _ . . . . . . . . . . 198

9 Providing Mixed-Language and Legacy Support in a Library: Experiences of Developing PETSc

Satish Balay, Jed Brown, Matthew Knepley, Lois Curfman McInnes, and Barry Smith

9.1 Introduction . . . . . . . . . . . . . . . . . 201

9.2 Fortran-C Interfacing Issues and Techniques . . . . . . . . 202

9.3 Automatically Generated Fortran Capability . . . . . . . . . 213

9.4 Conclusion . . . . . . . . . . . . . . . . . . . . . . 214

10 HydroShare - A Case Study of the Application of Modern Software Engineering to a Large Distributed Federally-Funded Scientific Software Development Project

Ray Idaszak, David G. Tarboton (Principal Investigator), Hong Yi, Laura Christopherson, Michael J. Stealey, Brian Miles, Pabitra Dash, Alva Couch, Calvin Spealman, Jeffery S. Horsburgh, and Daniel P. Ames 10.1 Introduction to HydroShare . . . . . . . . . . . . . 218

10.2 Informing the Need for Software Engineering Best Practices for

Science . . . . . . . . . . . . . . 220

10.3 Challenges Faced and Lessons Learned _ . . . . . . . . . 221

10.3.1 Cultural and Technical Challenges . . . . . . . . . . . 221

10.3.2 Waiting Too Long between Code Merges . . . . . . . . 223

10.3.3 Establishing a Development Environment . . . . . . . 224

10.4 Adopted Approach to Software Development Based on the Lessons Learned . . . . . . . . . . . . . . . . . . . 224

10.4.1 Adopting Best Practices in Modern Software

Engineering . . . . . . . . . . . . . . . 2 225

10.4.2 Iterative Software Development . . . . . . . . . . 226

10.4.3 Virtual Machines . . . . . . . . . . . . . . . . . . 227

10.4.4 Code Versioning . . . . . . . . . . . . . . . . . . 228

10.4.5 Code Reviews . . . . . . . . . . . . . . . . . . . 228

10.4.6 Testing and Test-Driven Development . . . . . . . . 229

10.4.7 Team Communication . . . . . . . . . . . . . . 229

10.4 .8 DevOps . . . . . . . . . . . . . . . 230 
10.5 Making Software Engineering More Feasible and Easier to Integrate into One's Research Activities . . . . . . . . . . . 231

10.6 Conclusion .................... . . . 232

\section{References}

Index 
- 


\section{List of Figures}

1.1 Development cycle of modeling with partial differential

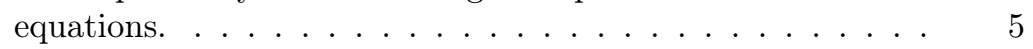

2.1 Overview of recommended process for documentation. . . . 32

2.2 SRS table of contents. . . . . . . . . . . . . 34

2.3 Proposed V\&V plan table of contents. . . . . . . . . . . . . 37

2.4 Proposed MG table of contents. . . . . . . . . . . . . 39

2.5 Example literate code documentation. . . . . . . . . . 40

2.6 Solar water heating tank, with heat flux $q_{c}$ from coil and $q_{P}$ to the PCM. . . . . . . . . . . . . . . . . . . . . . 42

2.7 Goal statements for SWHS. . . . . . . . . . . . . . . 43

2.8 Sample assumptions for SWHS. . . . . . . . . . . . . . 44

2.9 Sample theoretical model. . . . . . . . . . . . . . . 45

2.10 Sample general definition. . . . . . . . . . . . . . . 46

2.11 Sample instance model. . . . . . . . . . . . . . . . . . 47

2.12 Uses hierarchy among modules. . . . . . . . . . . . . . 48

3.1 Some challenges in testing the software. . . . . . . . . . 57

3.2 Program languages used in the Department of Plant Sciences. 59

3.3 Software engineering techniques used in the department. . . 60

3.4 SEIR model schematic. . . . . . . . . . . . . . . 67

3.5 Typical SEIR graph. . . . . . . . . . . . . . . . 68

3.6 Unexpected complexities of the model. . . . . . . . . . . 72

3.7 Unexpected complexities of the implementation. . . . . . . 74

3.8 The effect of tolerance thresholds. . . . . . . . . . . 75

3.9 Model schematic for HLB. . . . . . . . . . . . . . . . . 79

3.10 Two differences identified between M1 and M2. . . . . . 85

4.1 Trilinos dashboard. . . . . . . . . . . . . . . . . 115

5.1 Schematic of the relationship between the three regression tester tasks. . . . . . . . . . . . . . . . . 128

6.1 The major software component and workflow of the ACME Land Model ALM functional testing. . . . . . . . . . . . 138 
6.2 Cube visualization showing the call-tree of a three-day ACME simulation running on 32 nodes (508 cores) of the Titan machine. . . . . . . . . . . . . . . . . . . . 142

6.3 Vampir's trace visualization showing a three-day ACME simulation running on 32 nodes (508 cores) of the Titan machine. . . . . . . . . . . . . . . . .

6.4 The software function call within ALM. Each node represents a software function call. . . . . . . . . . . . . . . . 145

6.5 Overview of the ALM unit testing platform. . . . . . . . 146

7.1 Function from the SAXS project described in Section 7.5.1 used for calculating the radius of gyration of a molecule. . .

7.2 JUnit test case that uses the permutative MR to test the function in Figure 7.1. . . . . . . . . . . . . . . 156

7.3 Function for finding the maximum element in an array. . . . 158

7.4 Function for finding the average of an array of numbers. . . 158

7.5 Function for calculating the running difference of the elements in an array. . . . . . . . . . . . . . . . 158

7.6 CFGs for the functions max, average, and calcRun. . . . . 159

7.7 Overview of the approach. . . . . . . . . . . . 160

7.8 Function for calculating the sum of elements in an array. . . 161

7.9 Graph representation of the function in Figure 7.8. . . . . . 161

7.10 Random walk kernel computation for the graphs $G_{1}$ and $G_{2} . \quad 163$

7.11 Effectiveness of MRpred in predicting MRs. . . . . . . . . . 164

7.12 A faulty mutant produced by $\mu$ Java. . . . . . . . . . . 168

7.13 Overall fault detection effectiveness. . . . . . . . . . . . . . 169

7.14 Fault detection effectiveness across MRs. . . . . . . . . . . 169

7.15 Fault detection effectiveness across MRs. . . . . . . . . . 170

7.16 Fault detection effectiveness of multiple MRs. . . . . . . . . 170

7.17 Fault detection effectiveness across different fault categories. 171

7.18 Fault detection effectiveness across fault categories for individual MRs. . . . . . . . . . . . . . . . . . . 172

8.1 Usage relations in the layered architecture of scientific simulation software. . . . . . . . . . . . . . 180

8.2 Horizontal integration of multiple DSLs. . . . . . . . . . . 182

8.3 Multiple layers acting as domain-specific platforms for each other. . . . . . . . . . . . . . . . . . 183

8.4 DSL hierarchy for the Sprat Marine Ecosystem Model. . . . 184

8.5 Meta-model for the concept of Domain-Specific Language (DSL) hierarchies. . . . . . . . . . . . . . . . . 186

8.6 Engineering process of the Sprat approach. . . . . . . . . 187

8.7 IDE for the Sprat Ecosystem DSL. . . . . . . . . . . 193 


\section{List of Tables}

2.1 Improving Scientific Software Qualities via Rational Design 29

2.2 Recommended Documentation . . . . . . . . . . . . 31

2.3 Excerpt from Table of Input Variables for SWHS . . . . . . 45

3.1 Model Parameters Used to Find Differences . . . . . . . . . 83

3.2 p-Values Used to Find Differences . . . . . . . . . . . . . 84

3.3 Comparison of Areas under p-Value Progress Curves for the Search-Based Technique and Random Testing . . . . . . 86

3.4 Comparison of p-Values Achieved after 1 Hour for the SearchBased Technique and Random Testing . . . . . . . . 86

$5.1 \quad$ Detected Code Faults Classified by Severity . . . . . . . . 127

7.1 Functions Used in the Experiment . . . . . . . . . . . 166

7.2 Details of the Code Corpus . . . . . . . . . . . . . 167

7.3 The Metamorphic Relations Used in This Study . . . . . . . 167

7.4 Categories of Mutations in $\mu$ Java . . . . . . . . . . 168 
- 


\section{About the Editors}

Dr. Jeffrey C. Carver is an associate professor in the Department of Computer Science at the University of Alabama. Prior to his position at the University of Alabama, he was an assistant professor in the Department of Computer Science at Mississippi State University. He earned his $\mathrm{PhD}$ in computer science from the University of Maryland. His main research interests include software engineering for science, empirical software engineering, software quality, human factors in software engineering, and software process improvement. He is the primary organizer of the workshop series on Software Engineering for Science (http://www.SE4Science.org/workshops). He is a Senior Member of the IEEE Computer Society and a Senior Member of the ACM. Contact him at carver@cs.ua.edu.

Neil P. Chue Hong is director of the Software Sustainability Institute at the University of Edinburgh, which works to enable the continued improvement and impact of research software. Prior to this he was director of OMII-UK at the University of Southampton, which provided and supported free, opensource software for the UK e-Research community. He has a masters degree in computational physics from the University of Edinburgh and previously worked at Edinburgh Parallel Computing Centre as a principal consultant and project manager on data integration projects. His research interests include barriers and incentives in research software ecosystems and the role of software as a research object. He is the editor-in-chief of the Journal of Open Research Software and chair of the Software Carpentry Foundation Advisory Council. Contact him at N.ChueHong@software.ac.uk.

George K. Thiruvathukal is a professor of computer science at Loyola University Chicago and visiting faculty at Argonne National Laboratory in the Math and Computer Science Division and the Argonne Leadership Computing Facility. His research interests include parallel and distributed systems, software engineering, programming languages, operating systems, digital humanities, computational science, computing education, and broadening participation in computer science. His current research is focused on software metrics in open-source mathematical and scientific software. Professor Thiruvathukal is a member of the IEEE, IEEE Computer Society, and ACM. 
- 


\section{List of Contributors}

Daniel P. Ames

Department of Civil \&

Environmental Engineering

Brigham Young University

Provo, UT, USA

Katie Antypas

National Energy Research Scientific

Computing Center

Lawrence Berkeley National

Laboratory

Berkeley, CA, USA

\section{Satish Balay}

Mathematics and Computer Science

$$
\text { Division }
$$

Argonne National Laboratory

Argonne, IL, USA

Roscoe A. Bartlett

Sandia National Laboratories

Albuquerque, NM, USA

\section{Jed Brown}

Department of Computer Science

University of Colorado Boulder

Boulder, CO, USA

\section{Laura Christopherson}

RENCI

University of North Carolina at Chapel Hill

Chapel Hill, NC, USA

\section{Ethan Coon}

Computational Earth Sciences

Los Alamos National Laboratory

Los Alamos, NM, USA

\author{
Alva Couch \\ Department of Computer Science \\ Tufts University \\ Medford, MA, USA \\ Pabitra Dash \\ Utah State University \\ Logan, UT, USA
}

\section{Anshu Dubey}

Mathematics and Computer Science Division

Argonne National Laboratory

Argonne, IL, USA

\section{Daniel Hook}

Software Group

ESG Solutions

Kingston, ON, Canada

Jeffery S. Horsburgh

Department of Civil \&

Environmental Engineering

Utah State University

Logan, UT, USA

\section{Ray Idaszak}

RENCI

University of North Carolina at

Chapel Hill

Chapel Hill, NC, USA

\section{Arne N. Johanson}

Department of Computer Science

Kiel University

Kiel, Germany 
Upulee Kanewala

Computer Science Department

Montana State University

Bozeman, MT, USA

\section{Matthew Knepley}

Department of Computational \& Applied Mathematics

Rice University

Houston, TX, USA

\section{Xiaoye Sherry Li}

Computational Research Division

Lawrence Berkeley National

Laboratory

Berkeley, CA, USA

\section{Lois Curfman McInnes}

Mathematics and Computer Science Division

Argonne National Laboratory

Argonne, IL, USA

\section{Brian Miles}

CGI Group Inc.

Fairfax, VA, USA

\section{J. David Moulton}

Mathematical Modeling and Analysis Los Alamos National Laboratory

Los Alamos, NM, USA

\section{Andreas Oschlies}

GEOMAR Helmholtz Centre for

Ocean Research

Kiel, Germany

\section{Matthew Patrick}

Department of Plant Sciences

University of Cambridge

Cambridge, United Kingdom
Katherine Riley

Argonne Leadership Computing Facility

Argonne National Laboratory

Lemont, IL, USA

\section{Barry Smith}

Mathematics and Computer Science Division

Argonne National Laboratory

Argonne, IL, USA

\section{Spencer Smith}

Computing and Software

Department

McMaster University

Hamilton, ON, Canada

\section{Calvin Spealman}

Caktus Consulting Group, LLC

Durham, NC, USA

\section{Michael Stealey}

RENCI

University of North Carolina at Chapel Hill

Chapel Hill, NC, USA

\section{David G. Tarboton}

Department of Civil \&

Environmental Engineering

Utah State University

Logan, UT, USA

\section{Dali Wang}

Climate Change Science Institute

Oak Ridge National Laboratory

Oak Ridge, TN, USA

James M. Willenbring

Sandia National Laboratories

Albuquerque, NM, USA 
Frank Winkler

National Center for Computational Sciences

Oak Ridge National Laboratory

Oak Ridge, TN, USA

\section{Boris Worm}

Biology Department

Dalhousie University

Halifax, NS, Canada

Ulrike Meier Yang

Center for Applied Scientific Computing

Lawrence Livermore National

$$
\text { Laboratory }
$$

Livermore, CA, USA

\section{Zhuo Yao}

Department of Electrical Engineering \& Computer Science

University of Tennessee

Knoxville, TN, USA

\section{Hong Yi}

RENCI

University of North Carolina at Chapel Hill

Chapel Hill, NC, USA 
- 


\section{Acknowledgments}

Jeffrey C. Carver was partially supported by grants 1243887 and 1445344 from the National Science Foundation. Any opinions, findings, and conclusions or recommendations expressed in this material are those of the author(s) and do not necessarily reflect the views of the National Science Foundation.

Neil P. Chue Hong was supported by the UK Engineering and Physical Sciences Research Council (EPSRC) Grant EP/H043160/1 and EPSRC, BBSRC and ESRC Grant EP/N006410/1 for the UK Software Sustainability Institute.

George K. Thiruvathukal was partially supported by grant 1445347 from the National Science Foundation. Any opinions, findings, and conclusions or recommendations expressed in this material are those of the author(s) and do not necessarily reflect the views of the National Science Foundation.

MATLAB ${ }^{\circledR}$ is a registered trademark of The MathWorks, Inc. For product information, please contact:

The MathWorks, Inc.

3 Apple Hill Drive

Natick, MA 01760-2098 USA

Tel: 5086477000

Fax: 508-647-7001

E-mail: info@mathworks.com

Web: www.mathworks.com 
- 


\section{Introduction}

\section{General Overview}

Scientific software is a special class of software that includes software developed to support various scientific endeavors that would be difficult, or impossible, to perform experimentally or without computational support. Included in this class of software are, at least, the following:

- Software that solves complex computationally- or data-intensive problems, ranging from large, parallel simulations of physical phenomena run on HPC machines, to smaller simulations developed and used by groups of scientists or engineers on a desktop machine or small cluster

- Applications that support scientific research and experiments, including systems that manage large data sets

- Systems that provide infrastructure support, e.g. messaging middleware, scheduling software

- Libraries for mathematical and scientific programming, e.g. linear algebra and symbolic computing

The development of scientific software differs significantly from the development of more traditional business information systems, from which many software engineering best practices and tools have been drawn. These differences appear at various phases of the software lifecycle as outlined below:

- Requirements:

- Risks due to the exploration of relatively unknown scientific/engineering phenomena

- Risks due to essential (inherent) domain complexity

- Constant change as new information is gathered, e.g. results of a simulation inform domain understanding

- Design

- Data dependencies within the software 
- The need to identify the most appropriate parallelization strategy for scientific software algorithms

- The presence of complex communication or I/O patterns that could degrade performance

- The need for fault tolerance and task migration mechanisms to mitigate the need to restart time-consuming, parallel computations due to software or hardware errors

- Coding

- Highly specialized skill set required in numerical algorithms and systems (to squeeze out performance)

- Validation and Verification

- Results are often unknown when exploring novel science or engineering areas and algorithms

- Popular software engineering tools often do not work on the architectures used in computational science and engineering

- Deployment

- Larger node and core sizes coupled with long runtimes result in increased likelihood of failure of computing elements

- Long software lifespans necessitate porting across multiple platforms

In addition to the challenges presented by these methodological differences, scientific software development also faces people-related challenges. First, educational institutions teach students high-level languages and programming techniques. As a result, there is a lack of developers with knowledge of relevant languages, like Fortran, or low-level skills to handle tasks like code optimization. Second, the dearth of interdisciplinary computational science programs is reducing the pipeline of graduates who have the experience required to be effective in the scientific software domain. Furthermore, the lack of these programs is reducing the motivation for graduates to pursue careers in scientific software. Third, the knowledge, skills, and incentives present in scientific software development differ from those present in traditional software domains. For example, scientific developers may lack formal software engineering training, trained software engineers may lack the required depth of understanding of the science domain, and the incentives in the science domain focus on timely scientific results rather than more traditional software quality/productivity goals.

The continuing increase in the importance and prevalence of software developed in support of science motivates the need to better understand how software engineering is and should be practiced. Specifically, there is a need to understand which software engineering practices are effective for scientific 
software and which are not. Some of the ineffective practices may need further refinements to fit within the scientific context. To increase our collective understanding of software engineering for science, this book consists of a collection of peer-reviewed chapters that describe experiences with applying software engineering practices to the development of scientific software.

Publications regarding this topic have seen growth in recent years as evidenced by the ongoing Software Engineering for Science workshop series $^{1}[1-5]$, workshops on software development as part of the IEEE International Conference on eScience ${ }^{2,3}$ conference, and case studies submitted to the Working towards Sustainable Scientific Software: Practice and Experiences workshop series ${ }^{4,5}$. Books such as Practical Computing for Biologists [6] and Effective Computation in Physics [8] have introduced the application of software engineering techniques to scientific domains. In 2014, Nature launched a new section, Nature Toolbox ${ }^{6}$, which includes substantial coverage of software engineering issues in research. In addition, this topic has been a longstanding one in Computing in Science and Engineering $(\mathrm{CiSE})^{7}$, which sits at the intersection of computer science and complex scientific domains, notably physics, chemistry, biology, and engineering. CiSE also has recently introduced a Software Engineering Track to more explicitly focus on these types of issues ${ }^{8}$. EduPar is an education effort aimed at developing the specialized skill set (in concurrent, parallel, and distributed computing) needed for scientific software development $[7]^{9}$.

In terms of funding, the United States Department of Energy funded the Interoperable Design of Extreme-Scale Application Software (IDEAS) project $^{10}$. The goal of IDEAS is to improve scientific productivity of extremescale science through the use of appropriate software engineering practices.

\section{Overview of Book Contents}

We prepared this book by selecting the set of chapter proposals submitted in response to an open solicitation that fit with an overall vision for the book.

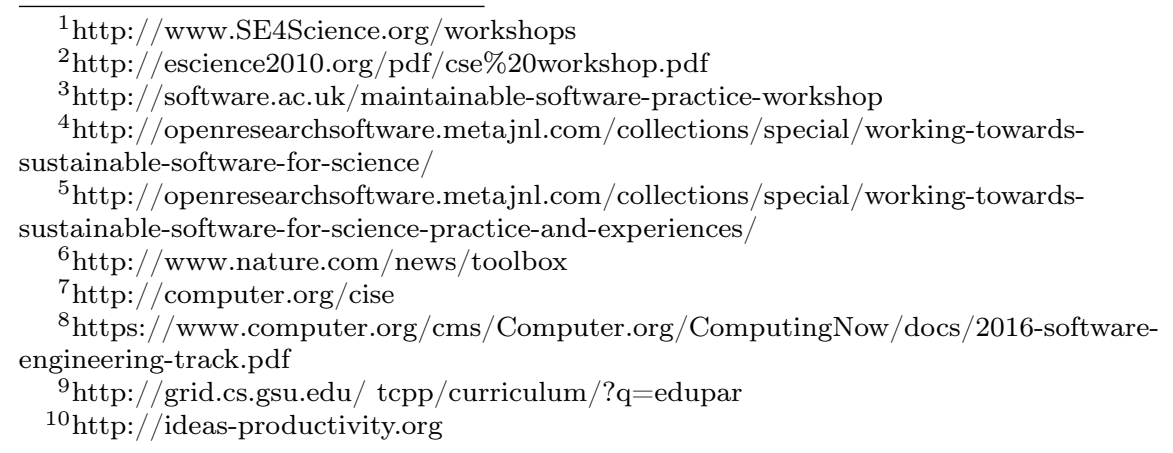


The chapters underwent peer review from the editors and authors of other chapters to ensure quality and consistency.

The chapters in this book are designed to be self-contained. That is, readers can begin reading whichever chapter(s) are interesting without reading the prior chapters. In some cases, chapters have pointers to more detailed information located elsewhere in the book. That said, Chapter 1 does provide a detailed overview of the Scientific Software lifecycle. To group relevant material, we organized the book into three sections. Please note that the ideas expressed in the chapters do not necessarily reflect our own ideas. As this book focuses on documenting the current state of software engineering in scientific software development, we provide an unvarnished treatment of lessons learned from a diverse set of projects.

\section{General Software Engineering}

This section provides a general overview of the scientific software development process. The authors of chapters in this section highlight key issues commonly arising during scientific software development. The chapters then describe solutions to those problems. This section includes three chapters.

Chapter 1, Software Process for Multiphysics Multicomponent Codes provides an overview of the scientific software lifecycle, including a number of common challenges faced by scientific software developers (note readers not interested in the full chapter may find this section interesting). The chapter describes how two projects, the long-running FLASH and newer Amanzi, faced a specific set of these challenges: software architecture and modularization, design of a testing regime, unique documentation needs and challenges, and the tension between intellectual property and open science. The lessons learned from these projects should be of interest to scientific software developers.

Chapter 2, A Rational Document Driven Design Process for Scientific Software argues for the feasibility and benefit of using a set of documentation drawn from the waterfall development model to guide the development of scientific software. The chapter first addresses the common arguments that scientific software cannot use such a structured process. Then the chapter explains which artifacts developers can find useful when developing scientific software. Finally, the chapter illustrates the document driven approach with a small example.

Chapter 3, Making Scientific Software Easier to Understand, Test, and Communicate through Software Engineering argues that the complexity of scientific software leads to difficulties in understanding, testing, and communication. To illustrate this point, the chapter describes three case studies from the domain of computational plant biology. The complexity of the underlying scientific processes and the uncertainty of the expected outputs makes adequately testing, understanding, and communicating the software a challenge. Scientists who lack formal software engineering training may find these 
challenges especially difficult. To alleviate these challenges, this chapter reinterprets two testing techniques to make them more intuitive for scientists.

\section{Software Testing}

This section provides examples of the use of testing in scientific software development. The authors of chapters in this section highlight key issues associated with testing and how those issues present particular challenges for scientific software development (e.g. test oracles). The chapters then describe solutions and case studies aimed at applying testing to scientific software development efforts. This section includes four chapters.

Chapter 4, Testing of Scientific Software: Impacts on Research Credibility, Development Productivity, Maturation, and Sustainability provides an overview of key testing terminology and explains an important guiding principle of software quality: understanding stakeholders/customers. The chapter argues for the importance of automated testing and describes the specific challenges presented by scientific software. Those challenges include testing floating point data, scalability, and the domain model. The chapter finishes with a discussion of test suite maintenance.

Chapter 5, Preserving Reproducibility through Regression Testing describes how the practice of regression testing can help developers ensure that results are repeatable as software changes over time. Regression testing is the practice of repeating previously successful tests to detect problems due to changes to the software. This chapter describes two key challenges faced when testing scientific software, the oracle problem (the lack of information about the expected output) and the tolerance problem (the acceptable level of uncertainty in the answer). The chapter then presents a case study to illustrate how regression testing can help developers address these challenges and develop software with reproducible results. The case study shows that without regression tests, faults would have been more costly.

Chapter 6, Building a Function Testing Platform for Complex Scientific Code describes an approach to better understand and modularize complex codes as well as generate functional testing for key software modules. The chapter defines a Function Unit as a specific scientific function, which may be implemented in one or more modules. The Function Unit Testing approach targets code for which unit tests are sparse and aims to facilitate and expedite validation and verification via computational experiments. To illustrate the usefulness of this approach, the chapter describes its application to the Terrestrial Land Model within the Accelerated Climate Modeling for Energy (ACME) project.

Chapter 7, Automated Metamorphic Testing of Scientific Software addresses one of the most challenging aspects of testing scientific software, i.e. the lack of test oracles. This chapter first provides an overview of the test oracle problem (which may be of interest even to readers who are not interested in the main focus of this chapter). The lack of test oracles, often resulting from 
the exploration of new science or the complexities of the expected results, leads to incomplete testing that may not reveal subtle errors. Metamorphic testing addresses this problem by developing test cases through metamorphic relations. A metamorphic relation specifies how a particular change to the input should change the output. The chapter describes a machine learning approach to automatically predict metamorphic relations which can then serve as test oracles. The chapter then illustrates the approach on several open source scientific programs as well as on in-house developed scientific code called SAXS.

\section{Experiences}

This section provides examples of applying software engineering techniques to scientific software. Scientific software encompasses not only computational modeling, but also software for data management and analysis, and libraries that support higher-level applications. In these chapters, the authors describe their experiences and lessons learned from developing complex scientific software in different domains. The challenges are both cultural and technical. The ability to communicate and diffuse knowledge is of primary importance. This section includes three chapters.

Chapter 8, Evaluating Hierarchical Domain-Specific Languages for Computational Science: Applying the Sprat Approach to a Marine Ecosystem Model examines the role of domain-specific languages for bridging the knowledge transfer gap between the computational sciences and software engineering. The chapter defines the Sprat approach, a hierarchical model in the field of marine ecosystem modeling. Then, the chapter illustrates how developers can implement scientific software utilizing a multi-layered model that enables a clear separation of concerns allowing scientists to contribute to the development of complex simulation software.

Chapter 9, Providing Mixed-Language and Legacy Support in a Library: Experiences of Developing PETSc summarizes the techniques developers employed to build the PETSc numerical library (written in C) to portably and efficiently support its use from modern and legacy versions of Fortran. The chapter provides concrete examples of solutions to challenges facing scientific software library maintainers who must support software written in legacy versions of programming languages.

Chapter 10, HydroShare - A Case Study of the Application of Modern Software Engineering to a Large, Distributed, Federally-Funded, Scientific Software Development Project presents a case study on the challenges of introducing software engineering best practices such as code versioning, continuous integration, and team communication into a typical scientific software development project. The chapter describes the challenges faced because of differing skill levels, cultural norms, and incentives along with the solutions developed by the project to diffuse knowledge and practice. 


\section{Key Chapter Takeaways}

The following list provides the key takeaways from each chapter. This list should help readers better understand which chapters will be most relevant to their situation. As stated earlier, the takeaways from each chapter are the opinions of the chapter authors and not necessarily of the editors.

\section{Chapter 1}

- The development lifecycle for scientific software must reflect stages that are not present in most other types of software, including model development, discretization, and numerical algorithm development.

- The requirements evolve during the development cycle because the requirements may themselves be the subject of the research.

- Modularizing multi-component software to achieve separation of concerns is an important task, but it difficult to achieve due to the monolithic nature of the software and the need for performance.

- The development of scientific software (especially multiphysics, multidomain software) is challenging because of the complexity of the underlying scientific domain, the interdisciplinary nature of the work, and other institutional and cultural challenges.

- Balancing continuous development with ongoing production requires open development with good contribution and distribution policies.

Chapter 2

- Use of a rational document-driven design process is feasible in scientific software, even if rational documentation has to be created post hoc to describe a development process that was not rational.

- Although the process can be time consuming, documenting requirements, design, testing and artifact traceability improves software quality (e.g., verifiability, usability, maintainability, reusability, understandability, and reproducibility).

- Developers can integrate existing software development tools for tasks like version control, issue tracking, unit testing, and documentation generation to reduce the burden of performing those tasks.

\section{Chapter 3}

- Scientific software is often difficult to test because it is used to answer new questions in experimental research. 
- Scientists are often unfamiliar with advanced software engineering techniques and do not have enough time to learn them, therefore we should describe software engineering techniques with concepts more familiar to scientists.

- Iterative hypothesis testing and search-based pseudo-oracles can be used to help scientists produce rigorous test suites in the face of a dearth of a priori information about its behavior.

\section{Chapter 4}

- The complexity of multiphysics scientific models and the presence of heterogeneous high-performance computers with complex memory hierarchies requires the development of complex software, which is increasingly difficult to test and maintain.

- Performing extensive software testing not only leads to software that delivers more correct results but also facilitates further development, refactoring, and portability.

- Developers can obtain quality tests by using granular tests at different levels of the software, e.g., fine-grained tests are foundational because they can be executed quickly and localize problems while higher-level tests ensure proper interaction of larger pieces of software.

- Use of an automated testing framework is critical for performing regular, possibly daily, testing to quickly uncover faults.

- Clearly defined testing roles and procedures are essential to sustain the viability of the software.

Chapter 5

- Use of regular, automated testing against historical results, e.g., regression testing, helps developers ensure reproducibility and helps prevent the introduction of faults during maintenance.

- Use of regression testing can help developers mitigate against the oracle problem (lack of information about the expected output) and the tolerance problem (level of uncertainty in the output).

\section{Chapter 6}

- The use of a scientific function testing platform with a compiler-based code analyzer and an automatic prototype platform can help developers test large-scale scientific software when unit tests are sparse.

- The function testing platform can help model developers and users better understand complex scientific code, modularize complex code, and generate comprehensive functional testing for complex code. 


\section{Chapter 7}

- The oracle problem poses a major challenge for conducting systematic automated testing of scientific software.

- Metamorphic testing can be used for automated testing of scientific software by checking whether the software behaves according to a set of metamorphic relations, which are relationships between multiple input and output pairs.

- When used in automated unit testing, a metamorphic testing approach is highly effective in detecting faults.

\section{Chapter 8}

- Scientists can use domain-specific languages (DSLs) to implement wellengineered software without extensive software engineering training.

- Integration of multiple DSLs from different domains can help scientists from different disciplines collaborate to implement complex and coupled simulation software.

- DSLs for scientists must have the following characteristics: appropriate level of abstraction for the meta-model, syntax that allows scientists to quickly experiment, have tool support, and provide working code examples as documentation.

\section{Chapter 9}

- Multi-language software, specifically Fortran, $\mathrm{C}$, and $\mathrm{C}++$, is still important and requires care on the part of library developers, benefitting from concrete guidance on how to call Fortran from $\mathrm{C} / \mathrm{C}++$ and how to call $\mathrm{C} / \mathrm{C}++$ from Fortran.

- Mapping of all common C-based constructs in multiple versions of Fortran allows developers to use different versions of Fortran in multilanguage software.

Chapter 10

- Use of modern software engineering practices helps increase the sustainability, quality and usefulness of large scientific projects, thereby enhancing the career of the responsible scientists.

- Use of modern software engineering practices enables software developers and research scientists to work together to make new and valuable contributions to the code base, especially from a broader community perspective.

- Use of modern software engineering practices on large projects increases the overall code capability and quality of science results by propagating these practices to a broader community, including students and postdoctoral researchers. 
- 